\title{
A New Method for Estimating Neutron Reaction Cross Sections Based on Wick's Limit
}

F. S. Dietrich, J. D. Anderson, R. W. Bauer, S. M. Grimes

October 18, 2004

International Conference on Nuclear Data for Science and Technology Santa Fe, NM, United States September 26, 2004 through October 1, 2004 
This document was prepared as an account of work sponsored by an agency of the United States Government. Neither the United States Government nor the University of California nor any of their employees, makes any warranty, express or implied, or assumes any legal liability or responsibility for the accuracy, completeness, or usefulness of any information, apparatus, product, or process disclosed, or represents that its use would not infringe privately owned rights. Reference herein to any specific commercial product, process, or service by trade name, trademark, manufacturer, or otherwise, does not necessarily constitute or imply its endorsement, recommendation, or favoring by the United States Government or the University of California. The views and opinions of authors expressed herein do not necessarily state or reflect those of the United States Government or the University of California, and shall not be used for advertising or product endorsement purposes. 


\title{
A New Method for Estimating Neutron Reaction Cross Sections Based on Wick's Limit
}

\author{
F. S. Dietrich*, J. D. Anderson*, R. W. Bauer* and S. M. Grimes ${ }^{\dagger}$ \\ *Lawrence Livermore National Laboratory, Livermore, CA 94550, USA \\ ${ }^{\dagger}$ Ohio University, Athens, OH 45701, USA
}

\begin{abstract}
Wick's limit is an inequality that relates the zero-degree differential elastic scattering cross section to the total cross section. The deviation of Wick's limit from an exact equality is small over a wide range of incident energies and target masses. Under these circumstances we show that Wick's limit can be used to correlate the uncertainties in the two terms of the reaction (nonelastic) cross section expressed as the difference between the total and angle-integrated elastic cross sections. When suitable elastic angular distributions are available, we show that the reaction cross section may be obtained with small errors (typically $1.5-3 \%$ ). Examples are shown for ${ }^{208} \mathrm{~Pb},{ }^{54-56} \mathrm{Fe},{ }^{232} \mathrm{Th}$, and ${ }^{238} \mathrm{U}$.
\end{abstract}

\section{WICK'S LIMIT}

Wick's limit [1] is derived from the optical theorem,

$$
\operatorname{Im} f\left(0^{\mathbf{o}}\right)=\frac{k}{4 \pi} \sigma_{t o t},
$$

where $f\left(0^{\mathrm{O}}\right)$ is the zero-degree scattering amplitude, $\sigma_{\text {tot }}$ is the total cross section, and $k$ is the center-of-mass wave number. The zero-degree differential elastic cross section $\sigma_{0}$ is given by

$$
\sigma_{0}=\left[\operatorname{Re} f\left(0^{\mathbf{O}}\right)\right]^{2}+\left[\operatorname{Im} f\left(0^{\mathbf{O}}\right)\right]^{2} \geq\left[\operatorname{Im} f\left(0^{\mathbf{O}}\right)\right]^{2} .
$$

Thus the zero-degree differential elastic cross section $\sigma_{0}$ must be equal to or exceed Wick's limit which is

$$
\sigma_{0}^{W} \equiv\left(\frac{k}{4 \pi} \sigma_{t o t}\right)^{2}
$$

We define the fractional deviation of the zero-degree differential cross section from Wick's limit as

$$
\eta=\frac{\sigma_{0}-\sigma_{0}^{W}}{\sigma_{0}^{W}}
$$

Wick's limit is a useful concept when $\eta$ is small. Fig. 1 shows the fractional deviation $\eta v s$. energy for several nuclei, using the global optical potential of Koning and Delaroche [2]. For all nuclei shown, there is a significant energy range (approximately 5-60 MeV) in which the deviation from Wick's limit does not exceed a few percent.

Insight into this behavior is provided by the Ramsauer model, a simple single-phase-shift model that reproduces

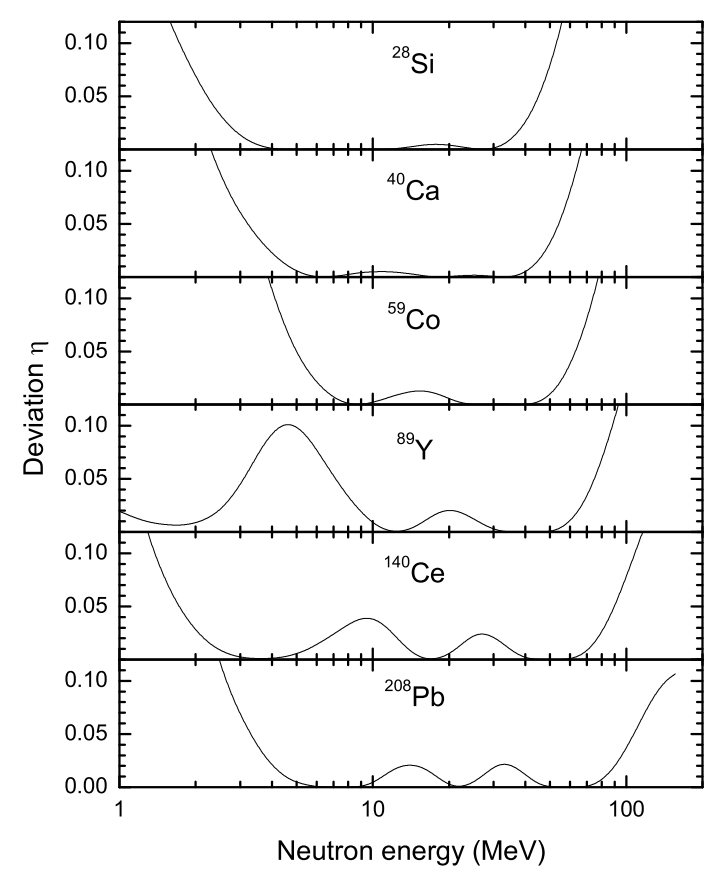

FIGURE 1. Fractional deviation $\eta$ using the optical potential of Ref. [2].

total cross sections at the $1 \%$ level from 5-60 MeV [3]. We define a quantity resembling an $S$-matrix element in terms of the forward scattering amplitude by

$$
S=1+\frac{2 i f\left(0^{\mathrm{O}}\right)}{k(R+\bar{\lambda})^{2}}
$$




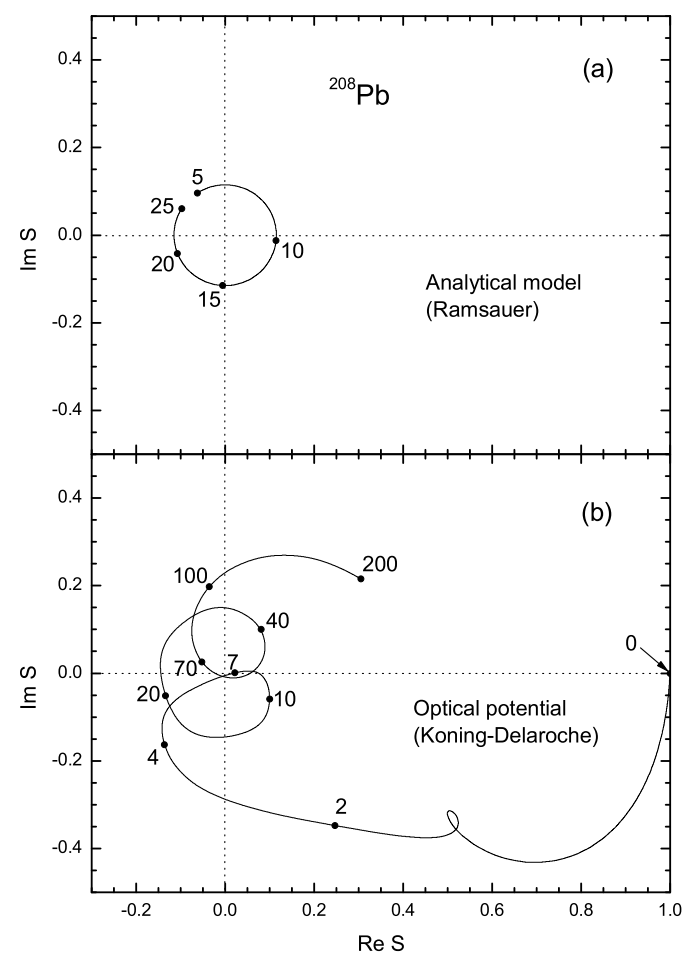

FIGURE 2. Single phase shift representation of the forward scattering amplitude for (a) the Ramsauer model and (b) a realistic optical model. Selected points on the trajectories are labeled by the incident energy in $\mathrm{MeV}$.

where $R=1.35 A^{1 / 3} \mathrm{fm}$, and examine the behavior of this quantity $v s$. energy in the complex plane, as shown for ${ }^{208} \mathrm{~Pb}$ in Fig. 2. Wick's limit is an equality (i.e. $\eta=0$ ) whenever $\operatorname{Im} S=0$. As seen in the upper part of the figure, the Ramsauer model has a trajectory that circles the origin. A realistic optical model [2] approximately reproduces this behavior for energies in the 4-90 MeV range, as indicated in the lower part of the figure, and shows that $\eta=0$ at several energies within this range.

\section{REACTION CROSS SECTION}

To estimate the reaction cross section, we begin with the expression

$$
\sigma_{\text {reac }}=\sigma_{\text {tot }}-\sigma_{\text {elas }},
$$

and define a quantity derived from the elastic scattering angular distribution that is independent of the absolute normalization of the cross section,

$$
F=\frac{\sigma_{\text {elas }}}{\sigma_{0}}=\frac{1}{\sigma_{0}} \int d \Omega \frac{d \sigma_{\text {elas }}}{d \Omega} .
$$

Inserting these in (6), using the definition of Wick's limit, we obtain the key result

$$
\sigma_{\text {reac }}=\sigma_{t o t}-(1+\eta) F\left(\frac{k}{4 \pi}\right)^{2} \sigma_{t o t}^{2}
$$

This expression yields the reaction cross section as a function of three independent quantities:

- $\sigma_{t o t}$, the total cross section;

- $F$, the ratio of angle-integrated to zero-degree elastic cross section;

- $\eta$, the fractional deviation of the zero-degree cross section from Wick's limit.

The fractional uncertainties in the reaction cross section, to be added in quadrature, are

$$
\begin{aligned}
\frac{\Delta \sigma_{\text {reac }}^{(1)}}{\sigma_{\text {reac }}} & =\left|2-\frac{\sigma_{\text {tot }}}{\sigma_{\text {reac }}}\right| \frac{\Delta \sigma_{\text {tot }}}{\sigma_{\text {tot }}}, \\
\frac{\Delta \sigma_{\text {reac }}^{(2)}}{\sigma_{\text {reac }}} & =\left(\frac{\sigma_{\text {tot }}}{\sigma_{\text {reac }}}-1\right) \frac{\eta}{1+\eta} \frac{\Delta \eta}{\eta}, \\
\frac{\Delta \sigma_{\text {reac }}^{(3)}}{\sigma_{\text {reac }}} & =\left(\frac{\sigma_{\text {tot }}}{\sigma_{\text {reac }}}-1\right) \frac{\Delta F}{F} .
\end{aligned}
$$

The error contribution from the total cross section is significantly reduced because of the correlation between the two terms of (8), and at certain energies (where $\sigma_{t o t}=2 \sigma_{\text {reac }}$ ) completely vanishes. The contribution from $F$ then becomes the dominant error, and its value depends on the accuracy with which measured angular distributions can be extrapolated to zero degrees via a Legendre expansion. The contribution from $\eta$ represents the accuracy with which $\eta$ can be obtained from an optical model; we assume a large value, typically $\Delta \eta / \eta=0.3$, for this model-dependent quantity.

\section{APPLICATIONS}

A first application of the method to reaction cross sections on ${ }^{208} \mathrm{~Pb}$ was presented in Ref. [3], using total cross sections from Ref. [4] and angular distributions from Ref. [5]. It was found that the uncertainties of approximately $5 \%$ in cross sections at 7, 20, 22, and $24 \mathrm{MeV}$ using direct subtraction via Eq. (6) were reduced to approximately $2 \%$. Results are shown in Fig. 3, along with an optical model calculation using the potential of Ref. [2]. Here we show new results for ${ }^{54,56} \mathrm{Fe}$ (we do not distinguish between ${ }^{56} \mathrm{Fe}$ and natural $\left.\mathrm{Fe}\right),{ }^{232} \mathrm{Th}$, and ${ }^{238} \mathrm{U}$.

Results for ${ }^{54} \mathrm{Fe}$ and ${ }^{56} \mathrm{Fe}$ are shown in the upper portion of Fig. 4. Total cross sections in the range 5.3$30 \mathrm{MeV}$ were taken from Ref. [6] with $1 \%$ uncertainty, and an extrapolation to $4 \mathrm{MeV}$ was made from entries 


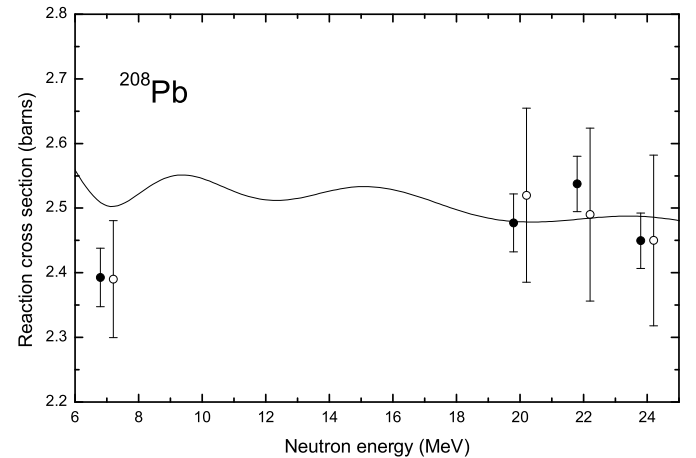

FIGURE 3. Reaction cross sections for ${ }^{208} \mathrm{~Pb}$ from present method (filled symbols) and by direct subtraction (open symbols). The energies of the points have been moved slightly for clarity. The curve is the optical model of Ref. [2].

in the CSISRS database. The quantity $F$ was calculated from Legendre expansions of the angular distributions indicated in the figure; angular distributions that did not yield stable behavior for $F$ with increasing maximum Legendre polynomial order were not included. The quantity $\eta$ was calculated with the optical potential of Ref. [2] with assumed uncertainty $\Delta \eta / \eta=0.3$. The results for ${ }^{54} \mathrm{Fe}$, indicated by open symbols, are slightly lower than the trend of the results for ${ }^{54} \mathrm{Fe}$, which is consistent with an approximate $A^{2 / 3}$ scaling. Data from the CSISRS database are shown in the lower part of the figure. The reaction cross section calculated from the optical potential of Ref. [2] approximately reproduces the energy dependence of the data, but its magnitude is slightly high.

The method may be generalized to deformed nuclei. To do this, we account for the fact that the measured angular distribution normally sums over several members of the ground-state band. The desired quantity is the compound nuclear cross section, defined as the reaction (nonelastic) cross section minus the cross section for direct excitation of the ground-state band members. The Wick deviation $\eta$ as well as corrections for contributions to the zero-degree cross section from excited states are estimated with a coupled-channel calculation. Results for ${ }^{232} \mathrm{Th}$ and ${ }^{238} \mathrm{U}$, using total cross sections from Ref. [6] and angular distributions from Ref. [7] are shown in Fig. 5. Also shown are cross sections for ${ }^{238} \mathrm{U}$ from the CSISRS database. The present results delineate the energy dependence of the cross section in the 4-10 MeV region with uncertainties in the neighborhood of $3 \%$.

\section{CONCLUSIONS}

The technique presented here can yield reaction cross sections with high precision $(\sim 1.5-3 \%)$ under suitable conditions:

- Angular distributions are available that allow reliable extrapolation to zero degrees. Experience from the results presented here suggests that the minimum angle should not exceed $20^{\circ}$ and that the number of points should exceed the maximum Legendre polynomial order by a factor approximately 1.5 .

- The optical model applies; i.e. energy fluctuations are small over the energy resolution of the angular distributions.

- The energy is in the range where deviations of the zero-degree cross section from Wick's limit are sufficiently small that the model dependence in the procedure is weak.

- The total cross sections employed must be accurately measured, even though the uncertainty propagating from the total cross section is significantly reduced in comparison with the direct-subtraction method.

Extension of the method to deformed nuclei is possible, as well as the ability to account for a small compound-elastic contribution (see Ref. [3]). Finally, we note that there are many additional angular distributions available to which the technique can be applied.

\section{ACKNOWLEDGMENTS}

This work was performed under the auspices of the U.S. Department of Energy by the University of California, Lawrence Livermore National Laboratory under contract No. W-7405-Eng-48, and Ohio University under contract No. DE-FG02-88ER40387.

\section{REFERENCES}

1. Wick, G. C., Phys. Rev., 75, 1459 (1949).

2. Koning, A. J., and Delaroche, J.-P., Nucl. Phys., A713, 231 (2003).

3. Dietrich, F. S., Anderson, J. D., Bauer, R. W., and Grimes, S. M., Phys. Rev. C, 68, 064608 (2003).

4. Finlay, R. W., Abfalterer, W. P., Fink, G., Montei, E., Adami, T., Lisowski, P. W., Morgan, G. L., and Haight, R. C., Phys. Rev. C, 47, 237 (1993).

5. Finlay, R. W., Annand, J. R. M., Cheema, T. S., Rapaport, J., and Dietrich, F. S., Phys. Rev. C, 30, 796 (1984).

6. Abfalterer, W. P., Bateman, F. B., Dietrich, F. S., Finlay, R. W., Haight, R. C., and Morgan, G. L., Phys. Rev. C, 63, 044608 (2001).

7. Smith, A. B., Nucl. Phys., A605, 269 (1996). 


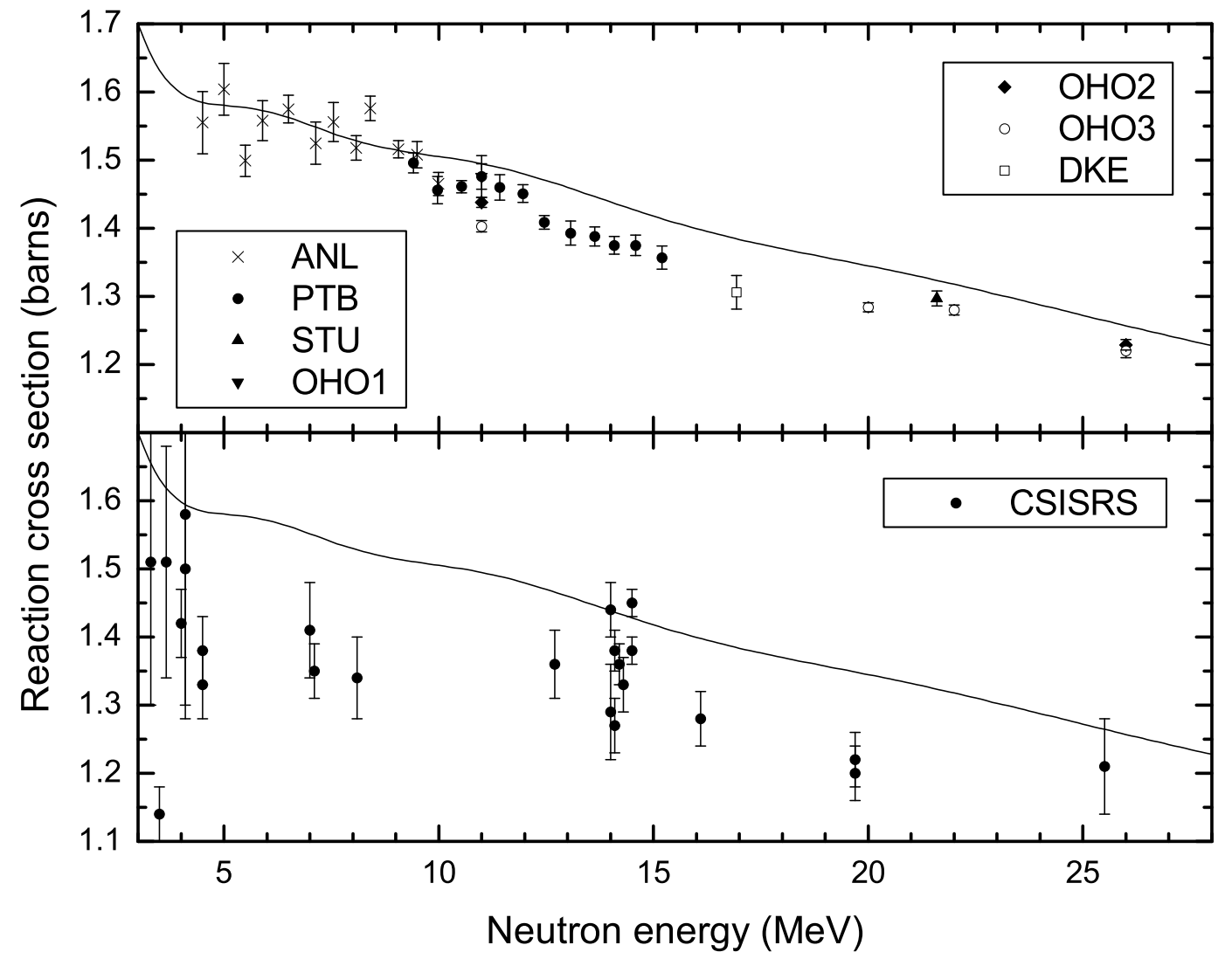

FIGURE 4. Reaction cross sections for ${ }^{54,56} \mathrm{Fe}$ from present method (upper portion) and CSISRS database (lower portion). Angular distributions are from Ref. [7] (ANL); Ref. [8] (PTB); Ref. [9] (STU); Ref. [10] (OHO1); Ref. [11] (OHO2); Ref. [11] (OHO3); and Ref. [12] (DKE). Open symbols are for ${ }^{54} \mathrm{Fe}$; the remainder for natural Fe. Curves are the optical model of Ref. [2].

8. Schmidt, D., Mannhart, W., Klein, H., and Nolte, R., Neutron scattering on natural iron at incident energies between 9.4 and 15.2 Mev, Tech. Rep. PTB-N-20, PTB, Braunschweig, Braunschweig, Germany (1994).

9. Olsson, N., Trostell, B., Ramström, E., Holmqvist, B., and Dietrich, F. S., Nucl. Phys., A472, 237 (1987).

10. Ferrer, J. C., Carlson, J. D., and Rapaport, J., Nucl. Phys., A275, 325 (1977).

11. Mellema, S., Finlay, R. W., Dietrich, F. S., and Petrovich, F., Phys. Rev. C, 28, 2267 (1983).

12. Pedroni, R. S., Howell, C. R., Honoré, G. M., Pfutzner, H. G., Byrd, R. C., Walter, R. L., and Delaroche, J. P., Phys. Rev. C, 38, 2052 (1988).

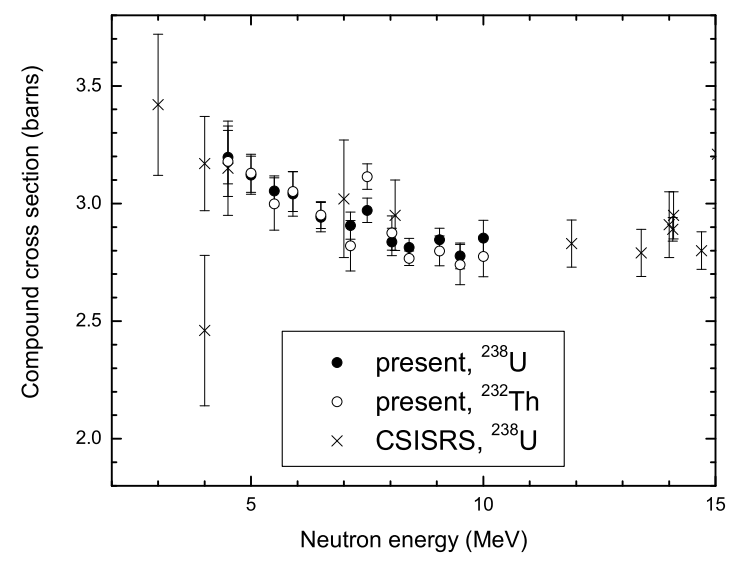

FIGURE 5. Compound nuclear cross section for ${ }^{232} \mathrm{Th}$ and ${ }^{238} \mathrm{U}$, calculated using angular distributions from Ref. [7]. Data from CSISRS database are also shown. 